\title{
Densidade mineral óssea de sítios específicos da maxila para a inserção de mini-implantes
}

Rodrigo César Santiago*, Giovanni Cerrone Júnior**, Robert Willer Farinazzo Vitral***

\begin{abstract}
Resumo
Objetivos: avaliar a Densidade Mineral Óssea (DMO) de sítios específicos na maxila, por meio da Tomografia Computadorizada (TC) Multi-Slice. Metodologia: foram realizadas 15 TC Multi-Slice da maxila para a avaliação da densidade mineral óssea de 30 regiões de interesse entre os segundos pré-molares e primeiros molares - 15 do lado direito e 15 do lado esquerdo - de 15 indivíduos ( 7 homens e 8 mulheres, com idade média de 21 anos) a serem submetidos ao tratamento ortodôntico, com mini-implantes como unidades de ancoragem. Resultados: o resultado do teste $t$ para observações pareadas revelou uma diferença estatisticamente significativa, quando os lados direito e esquerdo foram comparados $(\mathrm{p}<0,05)$. Conclusão: a média dos valores obtidos para a DMO encontrou-se próxima ao valor máximo de uma escala considerada normal para a região posterior da maxila, podendo, assim, ser considerada essa uma área segura em termos de qualidade óssea para a inserção de mini-implantes.
\end{abstract}

Palavras-chave: TC Multi-Slice. Densidade óssea. Maxila.

\section{INTRODUÇÃO}

Dentre os vários sistemas desenvolvidos para permitir o movimento distal de caninos nos casos com extração de pré-molares ${ }^{8,11,25,28,29}$, os mini-implantes constituem um mecanismo de ancoragem temporária, com vantagens como a ausência da necessidade de cooperação por parte dos pacientes em relação aos demais sistemas de ancoragem convencionais, a redução do tempo do tratamento ${ }^{5,15,16}$, a capacidade de suportar uma variedade de forças ortodônticas envolvendo pacientes com dentição mutilada ou nos casos de extração que requerem uma ancoragem máxima ${ }^{16}$, fácil inserção e remoção, baixo custo e possibilidade de ativação imediata ${ }^{6}$.
Apesar de estudos descreverem a eficácia e a estabilidade deste novo modelo de ancoragem nos tratamentos das más oclusões ${ }^{15,18,20,22,24-27}$, o comportamento clínico dos mini-implantes sob ação de cargas ortodônticas ainda não está totalmente claro. A questão se eles se mantêm absolutamente estáveis como os implantes endósseos ou se podem sofrer deslocamento à medida que a força ortodôntica é aplicada permanece sem resposta ${ }^{15}$.

Pesquisas demonstram a importância de se obter um conhecimento prévio da densidade óssea para um correto planejamento e execução de implantes dentários convencionais ${ }^{1,3,4,19}$, onde altas taxas de fracasso têm sido associadas a implantes realizados em osso de qualidade baixa.

\footnotetext{
* Especialista em Ortodontia e mestre em Saúde/Grupo de Pesquisa em Ortodontia pela UFJF/MG.

** Professor do Curso de Especialização em Radiologia da FO-UFJF/MG.

*** Mestre e doutor em Ortodontia - UFRJ/RJ. Coordenador do curso de especialização em Ortodontia da FO-UFJF/MG.
} 
Estudos revelam ser a Tomografia Computadorizada Quantitativa um método eficaz para o estudo da Densidade Mineral Óssea (DMO). Uma das principais vantagens é que as informações resultantes não sofrem sobreposição por estruturas anatômicas adjacentes ${ }^{32}$. A alta sensibilidade na diferenciação entre os tipos de tecidos permite detectar diferenças de densidades de $1 \%$ ou menos ${ }^{9}$.

Uma escala preestabelecida por Norton e $\mathrm{Gamble}^{21}$ apresenta os valores quantitativos para a DMO, determinados em Unidades Hounsfiled $(\mathrm{HU})$, para quatro diferentes regiões dos maxilares: > +850 HU (região mandibular anterior); +500 até $+800 \mathrm{HU}$ (regiões mandibular posterior e maxilar anterior) e 0 até $+500 \mathrm{HU}$ (região maxilar posterior). Trabalhos que utilizaram a tomografia computadorizada quantitativa para correlacionar a DMO da mandíbula, avaliada no pré-operatório, e a intensidade da força de torque durante a inserção de implantes encontraram uma correlação positiva, concluindo que a determinação da DMO através da TC pode ser utilizada para se estimar a estabilidade primária desses implantes $2,7,10,13,30$.

O presente estudo teve como objetivo avaliar a DMO da região entre segundos pré-molares e primeiros molares da maxila, através de Tomografia Computadorizada Multi-Slice, com a finalidade de determinar, através da comparação com uma escala de densidade óssea da região posterior da maxila ${ }^{21}$, a segurança dessa região em termos de qualidade óssea para a inserção de mini-implantes.

\section{MATERIAL E MÉTODOS Seleção da amostra}

A amostra constou de 15 indivíduos (com idades variando entre 12 anos e 5 meses e 32 anos e 11 meses) selecionados consecutivamente para o início do tratamento na clínica de pós-graduação em Ortodontia da Faculdade de Odontologia da Universidade Federal de Juiz de Fora/MG, respeitando os seguintes critérios:

a) A idade mínima respeitou um limite de 12 anos de idade, sem, entretanto, estabelecer-se uma idade máxima limite. A idade mínima foi estabelecida conforme orientações da Agência Americana de Administração de Drogas e Alimentos (FDA/ EUA).

b) Presença de todos os dentes permanentes irrompidos, exceto os terceiros molares.

c) Ausência de tratamento ortodôntico e/ou ortopédico prévio.

d) Boas condições de higiene bucal.

e) Presença de má oclusão que justificasse a exodontia de primeiros pré-molares permanentes e necessitasse da distalização dos caninos.

f) Ausência de insuficiência renal crônica e distúrbios hormonais (especialmente distúrbios da tireoide, da paratireoide ou das adrenais).

g) Não usuários de drogas, como corticosteroides, barbitúricos, anticonvulsivantes e quantidades excessivas de hormônio tireoidiano.

h) Não alcoólatras e/ou tabagistas.

O trabalho foi aprovado pelo Comitê de Ética em Pesquisa da UFJF/MG, sob o parecer 214/2005.

\section{Avaliação da Densidade Mineral Óssea (DMO)}

Foram realizadas 15 Tomografias Computadorizadas Multi-Slice (Somaton Spirit ${ }^{\circledR}$, SIEMENS, Alemanha) da maxila. No momento da realização do exame, os indivíduos foram posicionados conforme padronizado por Vitral, Telles ${ }^{31}$ eVitral et al. ${ }^{32}$

Para a determinação da altura mínima na região de septo inter-radicular na região entre segundos pré-molares e primeiros molares superiores com quantidade de osso trabeculado suficiente para inserção dos mini-implantes, com no mínimo $2 \mathrm{~mm}$ de distância entre as corticais, foram realizadas duas radiografias periapicais, lados direito e esquerdo (técnica do paralelismo), nos 15 indivíduos da amostra.

$\mathrm{Na}$ elaboração do tomograma, utilizou-se o software Denta Scan (General Eletric - Medical Systems, EUA). Para a reconstrução paraxial, foram empregadas as seguintes medidas de 
referência: length $=16,0$; distance $=1,0$; thickness $=1,0 \mathrm{~mm}$. A reconstrução panorâmica (Fig. 1) permitiu a identificação do corte que representaria a região de septo inter-radicular para o estudo. Através da reconstrução paraxial (Fig. 2), identificou-se o local de inserção do mini-implante e delimitou-se a maior região de interesse (region of interest - ROI) possivel que abrangesse osso cortical vestibular e medular da região.

A determinação da Densidade Mineral Óssea por área $\left(\mathrm{cm}^{2}\right)$, em Unidades Hounsfield (HU), foi realizada com o software Denta Scan. Os valores encontrados expressaram a densidade média da área delimitada (ROI).

\section{Erro de método}

Para a avaliação do erro do método, foi utilizado o coeficiente de correlação intraclasse. $\mathrm{O}$ valor obtido foi de 0,994 .

\section{Análise estatística}

Utilizou-se o teste $t$ de Student pareado durante a avaliação das diferenças entre as médias obtidas do lado direito e esquerdo de cada indivíduo da amostra.

Com o objetivo de avaliar a correlação entre os lados direito e esquerdo, procedeu-se um teste de regressão linear. Para a análise do padrão de distribuição dos valores obtidos, foi utilizado o teste de normalidade Shapiro-Wilk.

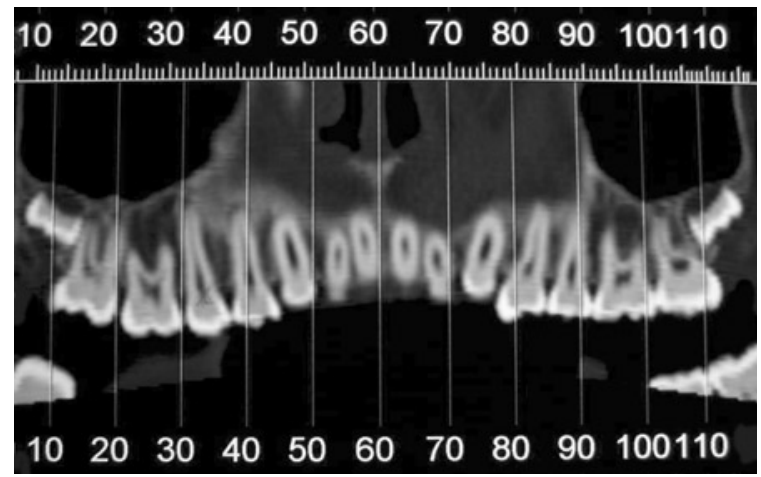

FIGURA 1 - Reconstrução panorâmica para identificação do corte referente ao local de inserção do mini-implante.

\section{RESULTADOS}

A tabela 1 apresenta os valores obtidos na avaliação da DMO (HU) das 30 regiões de interesse, nos lados direito e esquerdo.

$O$ resultado do teste $t$ de Student pareado entre os lados direito e esquerdo de cada elemento da amostra encontram-se da tabela 2. O valor de $\mathrm{P}$ encontrado foi de 0,045 .

A tabela 3 expressa o teste de regressão linear durante a avaliação da correlação entre os lados direito e esquerdo. O coeficiente de correlação foi igual a $0,44(\mathrm{p}=0,097)$.

A tabela 4 apresenta uma análise descritiva da distribuição das 30 médias de DMO. O teste de normalidade Shapiro-Wilk apresentou um valor de $\mathrm{p}=0,842$.

\section{DISCUSSÃO}

Vários estudos demonstram a eficácia dos mini-implantes como um dispositivo auxiliar de ancoragem no tratamento das más oclusões ${ }^{15,20,24-27} \mathrm{e}$ é reconhecido que a qualidade óssea do local de inserção é um dos fatores que podem interferir na estabilidade desses dispositivos ${ }^{15,18,20,24}$.

A tomografia computadorizada é um procedimento de rotina durante a determinação da qualidade óssea na realização de implantes convencionais endósseos ${ }^{2,7,10}$, entretanto, tal situação não é verificada na utilização dos mini-implantes com finalidade de ancoragem.

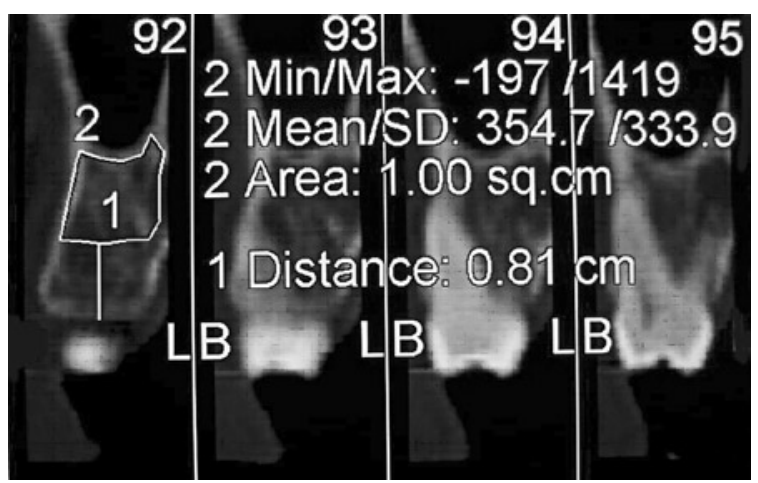

FIGURA 2 - Reconstrução paraxial para determinação da DMO no local de inserção do mini-implante. 
TABELA 1 - Valores da Densidade Mineral Óssea (HU).

\begin{tabular}{|c|c|c|c|c|c|c|c|c|c|c|c|c|c|c|c|}
\hline indivíduo & 1 & 2 & 3 & 4 & 5 & 6 & 7 & 8 & 9 & 10 & 11 & 12 & 13 & 14 & 15 \\
\hline lado direito & 507,5 & 486,1 & 480,2 & 385,0 & 512,1 & 497,6 & 448,6 & 397,8 & 407,8 & 498,7 & 409,5 & 369,5 & 660,8 & 346,6 & 373,3 \\
\hline lado esquerdo & 329,0 & 319,3 & 409,0 & 265,7 & 507,2 & 354,7 & 310,1 & 510,3 & 317,5 & 487,2 & 462,0 & 167,0 & 563,5 & 279,6 & 555,9 \\
\hline
\end{tabular}

TABELA 2 - Média e desvio-padrão da diferença entre as médias das diferentes regiões, distribuídas por lado (direito/esquerdo).

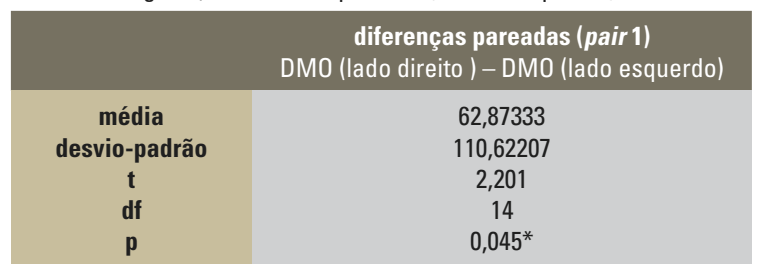

* estatisticamente significativo ao nível de $\mathrm{p}<0,05$.

TABELA 3 - Correlação entre amostras pareadas.

\begin{tabular}{|c|c|} 
& DMO (lado direito) $\mathbf{x}$ DMO (lado esquerdo) \\
\hline $\mathbf{n}$ & 15 \\
correlação & 0,444 \\
sig. & 0,097 \\
\hline
\end{tabular}

Em uma escala previamente estabelecida por Norton e Gamble ${ }^{21}$ para a DMO da região posterior da maxila, os valores variaram de $0 \mathrm{a}+500 \mathrm{HU}$. Para a amostra dessa pesquisa, os valores de DMO encontrados variaram de $+167 \mathrm{HU}$ a $+660,80 \mathrm{HU}$, com um valor médio de $+420,63 \mathrm{HU}$, sendo que a região específica entre os segundos pré-molares e os primeiros molares apresentou uma média próxima ao valor máximo da escala citada anteriormente, revelando ser essa uma região com qualidade significativa para essa amostra.

Ao se comparar a média das diferenças dos lados direito e esquerdo, diferença estatisticamente significativa foi observada através do teste $t$ para amostras pareadas, refletindo o achado em que, dos 15 casos estudados, 12 apresentaram a densidade mineral óssea do lado direito da maxila maior que a do lado esquerdo. Estudos revelaram que a existência de um lado preferencial de mastigação pode levar a alterações de densidade óssea
TABELA 4 - Análise descritiva da distribuição das 30 médias observadas (teste de normalidade de Shapiro-Wilk).

\begin{tabular}{|c|c|c|c|}
\hline \multicolumn{2}{|c|}{ descritivas } & & \multirow[t]{2}{*}{ estatística } \\
\hline 30 médias & $\begin{array}{l}\text { média }=420,6367 \\
\text { mediana }=409,2500 \\
\text { d.p. }=105,25056 \\
\text { mínimo }=167,00 \\
\text { máximo }=660,80\end{array}$ & & \\
\hline & Shapiro-Wilk & df & \multirow{2}{*}{$\mathrm{p}$} \\
\hline & estatística & 3 & \\
\hline 30 médias & 0,981 & 0 & 0,842 \\
\hline
\end{tabular}

na maxila e mandíbula $a^{17,23}$. O teste de correlação linear revelou, também, uma baixa correlação entre os dois lados analisados.

As médias de DMO avaliadas apresentaram uma distribuição normal, segundo o teste de normalidade Shapiro-Wilk. Os valores obtidos não apresentaram discrepâncias estatisticamente significativas em relação ao padrão observado no grupo.

No presente estudo, a idade média da amostra foi de 21 anos e 1 mês. Como há diminuição do metabolismo de cálcio com o aumento da idade, principalmente no gênero feminino ${ }^{12,14}$, variações devem ser esperadas ao se estudar amostras com uma média de idade maior que a do grupo avaliado nesse estudo, acrescentando que essas diferenças podem ter um papel relevante na estabilidade dos mini-implantes.

Clinicamente, os resultados desse estudo sugerem a realização de exames complementares, como a TC Multi-Slice, durante o planejamento do uso 
de mini-implantes, para que possa vir a constituir mais um instrumento utilizado pelo ortodontista durante a escolha do local mais seguro, em termos de qualidade óssea, para a inserção desses dispositivos de ancoragem.

\section{CONCLUSÕES}

- As médias de densidade óssea encontradas apresentaram uma distribuição normal, onde os valores obtidos não apresentaram discrepâncias estatisticamente significativas em relação ao padrão observado no grupo.

- Diferença estatisticamente significativa foi observada entre os lados direito e esquerdo.
- A média dos valores obtidos para a DMO encontrou-se próxima do valor máximo de uma escala considerada normal para a região posterior da maxila, podendo, assim, a região estudada ser considerada uma área segura, em termos de qualidade óssea, para a inserção de mini-implantes.

\title{
Bone mineral density of specific maxillary sites for mini-implants insertion
}

\begin{abstract}
Aim: To assess the Bone Mineral Density (BMD) of specific maxillary sites, through multi-slice $\mathrm{CT}$, investigating the possible correlation between different BMD levels and mini-implant stability. Methods: Scans were obtained from 30 regions of interest (ROI), 15 on the right side and 15 on the left side, of 15 individuals ( 7 males and 8 females with 21 years old mean age) about to start orthodontic treatment with mini-implants as anchorage units. BMD values were normally distributed. Paired t-test results showed statistically significant differences when the two sides were compared $(p<0.05)$. However, the mean of BMD values was close to the maximum value of a considered normal scale for maxillary posterior region, indicating that this is a safe area in terms of bone quality for mini-implants insertion.
\end{abstract}

Keywords: Multi-Slice CT. Bone density. Maxillary.

\section{REFERÊNCIAS}

1. ALBREKTSSON, T. On long-term maintenance of the osseointegrated response. Aust. Prosthodont. J., Westmead, v. 7, p. 15-24, 1993.

2. BEER, A.; GAHLEITNER, A.; HOLM, A.; TSCHABITSHER, M.; HOMOLKA, P. Correlation if insertion torques with bone mineral density from dental quantitative $\mathrm{CT}$ in the mandible. Clin. Oral Implants Res., Copenhagen, v. 14, no. 5, p. 616-620, Oct. 2003.

3. CUMMINGS, S. R.; BATES, D.; BLACK, D. M. Clinical use of bone densitometry: scientific review. JAMA, Chicago, v. 288, no. 15, p. 1889-1997, Oct. 2002.
4. EBBESEN, E. N.: THOMSEN, J. S.; BECK-NIELSEN, J.; NEPPER-RASMUSSEN, H. J.; MOSEKILDE, L. Vertebral bone density evaluated by dual-energy $\mathrm{x}$-ray absorptiometry and quantitative computed tomography in vitro. Bone, New York, v. 23, no. 3, p. 283-290, Sept. 1998.

5. HERMAN, R.; COPE, J. B. Miniscrew implants: IMTEC mini ortho implants. Semin. Orthod., Philadelphia, v. 11, no. 1, p. 32-39, Mar. 2005.

6. HEYMANN, G. C.; TULLOCH, J. F. Implantable devices as orthodontic anchorage: a review of current treatment modalities. J. Esthet. Restor. Dent., Hamilton, v. 18, no. 2, p. 68-79, Mar./Apr. 2006.

7. HOMOLKA, P.; BEER, A.; BIRKFELINER, W.; NOWOTNY, R.; GAHLEITNER, A.; TSCHABITSHER, M. et al. Bone mineral density measurement with dental quantitative $\mathrm{CT}$ prior to the dental implant placement in cadaver mandibles: pilot study. Radiology, Easton, v. 224, no. 1, p. 247-252, July 2002. 
8. HUANG, L. H. SHOTWELL, J. L. WANG, H. L. Dental implants for orthodontic anchorage. Am. J. Orthod. Dentofacial Orthop., St. Louis, v. 127, no. 6, p. 713-722, June 2005.

9. IBÁÑEZ, R. Bone mineral density measurement techniques. An. Sist. Sanit. Navar, Pamplona, v. 26, p. 19-27, Sept./Dec. 2003. Supplement 3.

10. IKUMI, N.; TSUTSUMI, S. Assessment of correlation between computerized tomography values of the bone and cutting torque values at implant placement: a clinical study. Int. J. Oral Maxillofac. Implants, Lombard, v. 20, no. 2, p. 253-260, Mar./ Apr. 2005.

11. ISMAIL, S. F. H.; JOHAL, A. S. Current products and practice section: the role of implants in Orthodontics. J. Orthod. London, v. 29, p. 239-245, Sept. 2002.

12. LAMBERG-ALLARDT, C. J.; OUTILA, T. A.; KARKKAINEN, M. U.; RITA, H. J.; VALSTA, L. M. Vitamin D deficiency and bone health in adults in Finland: could this be a concern in other parts of Europe? J. Bone Miner. Res., Washington, D.C., v. 16, no. 11, p. 2066-2073, Nov. 2001

13. LEKHOLM, U.; ZAHR, G. A. Patient selection and preparation. In: BRANEMARK, P. I.; ZARB, G. A.; ALBREKTSSON, T. (Org.). Osseointegration in clinical Dentistry. 1st ed. Chicago: Quintessence, 1985. p. 199-209.

14. LIN, J. T.; LANE, J. M. Osteoporosis: a review. Clin. Orthop. Relat. Res., Philadelphia, v. 425, no. 8, p. 126-134, Aug. 2004.

15. LIOU, E. J. W.; PAI, B. C. J.; LIN, J. C. Y. Do miniscrew remain stationary under orthodontic forces? Am. J. Orthod. Dentofacial Orthop., St. Louis, v. 126, no. 1, p. 42-47, July 2004.

16. MAINO, B. G.; MURA, P.; BEDNAR, J. Miniscrew implants: the spider screw anchorage system. Semin. Orthod., Philadelphia, v. 11, no. 1, p. 40-46, Mar. 2005.

17. McDONNELL, S. T.; HECTOR, M. P.; HANNIGAN, A. Chewing side preferences in children. J. Oral Rehabil., Oxford, v. 31, no. 9, p. 855-860, Sept. 2004.

18. MELSEN, B.; VERNA, C. Miniscrew implants: the Aarhus anchorage system. Semin. Orthod., Philadelphia, v. 11, no. 1, p. 24-31, Mar. 2005

19. MISCH, C. E. Divisions of available bone in implant Dentistry. Int. J. Oral Implantol., New York, v. 7, no. 1, p. 9-17, Jan. 1990

20. MIYAWAKI, S.; KOYAMA, I.; INOUE, M.; MISHIMA, K.; SUGAHARA, T.; TAKANO-YAMAMOTO, T. Factors associated with the stability of titanium screws placed in the posterior region for orthodontic anchorage. Am. J. Orthod. Dentofacial Orthop., St. Louis, v. 124, no. 4, p. 373-378, Oct. 2003.
21. NORTON, M. R.; GAMBLE, C. Bone classification: an objective scale of bone density using the computerized tomography scan. Clin. Oral Impl. Res., Copenhagen, v. 12, no. 1, p. 79-84, Feb. 2001.

22. OHNISHI, H.; YAGI, T.; YASUDA, Y.; TAKADA, K. A mini-implant for orthodontic anchorage in a deep overbite case. Angle Orthod., Appleton, v. 75, no. 3, p. 444-452, May 2005.

23. PAPHANGKORAKIT, J.; THOTHONGKAM, N.; SUPANONT, N. Chewing-side determination of three food textures. J. Oral Rehabil., Oxford, v. 33, no. 1, p. 2-7, Jan. 2006.

24. PARK, H. S.; JEONG, S. H.; KWON, O. W. Factors affecting the clinical success of screw implants used as orthodontic anchorage. Am. J. Orthod. Dentofacial Orthop., St. Louis, v. 130, no. 1, p. 18-25, July 2006.

25. PARK, H. S.; KWON, T. G. Sliding mechanics with microscrew implant anchorage. Angle Orthod., Appleton, v. 74, no. 5, p. 703-710, Sept. 2004.

26. PARK, H. S.; KWON, T. G.; SUNG, J. H. Nonextraction treatment with microscrew implants. Angle Orthod., Appleton, v. 74, no. 4, p. 539-549, July 2004.

27. PARK, H. S.; LEE, S. K.; KWON, O. W. Group distal movement of teeth using microscrew implant anchorage. Angle Orthod., Appleton, v. 75, no. 4, p. 602-609, July 2005.

28. RAJCICH, M. M.; SADOWSKY, C. Efficacy of intraarch mechanics using differential moments for achieving anchorage control in extraction cases. Am. J. Orthod. Dentofacial Orthop., St. Louis, v. 112, no. 4, p. 441-448, Oct. 1997.

29. SCHNELLE, M. A.; BECK, F. M.; JAYNES, R. M.; HUJA, S. S. Radiographic evaluation of the availability of bone for placement of miniscrews. Angle Orthod., Appleton, v. 74 no. 6, p. 832-837, Nov. 2004.

30. SHAHLAIE, M.; GANTES, B.; SCHULZ, E.; RIGGS, M. CRIGGER, M. Bone density assessment of dental bone sites: 1. quantitative computed tomography. Int. J. Oral Maxillofac. Implants, Lombard, v. 18, no. 2, p. 224-231, Mar./Apr. 2003.

31. VITRAL, R. W. F.; TELLES, C. S. Computed tomography evaluation of temporomandibular joint alterations in Class II Division 1 subdivision patients: condylar symmetry. Am. J. Orthod. Dentofacial Orthop., St. Louis, v. 121, no. 4, p. 369-375, Apr. 2002.

32. VITRAL, R. W. F.; TELLES, C. S.; FRAGA, M. R.; OLIVEIRA R. S. F.; TANAKA, O. M. Computed tomography evaluation of temporomandibular joint alterations in patients with Class II division 1 subdivision malocclusions: condyle-fossa relationship. Am. J. Orthod. Dentofacial Orthop., St. Louis, v. 126, no. 1, p. 48-52, July 2004.
Endereço para correspondência

Rodrigo César Santiago

Rua Tiradentes 510/802, Santa Helena

CEP: 36.015-360 - Juiz de Fora / MG

E-mail: rodrigo_cesar_santiago@hotmail.com 